\title{
HTPCS: revelando los obstáculos de la función del coordinador pedagógico durante el proceso de formación continua del profesorado
}

\author{
HTPCs: Revealing the obstacles of the performance of pedagogical coordinator \\ during the process of continuing training of teachers
}

\author{
José Pedro Lino \\ jpedro.lino@gmail.com \\ Universidad Nacional de La Plata, Argentina
}

Recepción: 02 Febrero 2020

Aprobación: 18 Marzo 2021

Publicación: 01 Abril 2021

Cita sugerida: Lino, J. P. (2020). HTPCS: revelando los obstáculos de la función del coordinador pedagógico durante el proceso de formación continua del profesorado. Archivos de Ciencias de la Educación, 14(18), e091. https://doi.org/10.24215/23468866e091
Resumen: Este artículo expone algunos resultados de la investigación de máster que tuvo como objetivo analizar las limitaciones, oportunidades y desafíos relacionados con el desempeño del coordinador pedagógico como agente articulador en la formación continua de los maestros de escuelas primarias. El artículo, además de proporcionar definiciones de la función del coordinador pedagógico, analiza cómo este profesional actúa en la implementación de procesos de formación desarrollados en las Horas de Trabajo Pedagógico Colectivo (HTPCs). En este contexto, se hace énfasis en que la trayectoria de la actuación del coordinador pedagógico está llena de obstáculos y que el sistema educativo tiene defectos que aún impactan en el trabajo pedagógico de este profesional. El camino de la investigación metodológica implicó estudios descriptivos y exploratorios, con el fin de comprender la realidad de la actuación de la Coordinación Pedagógica en las escuelas establecidas para la investigación de campo, con respecto a su regimiento y papel práctico-teórico. En base a los datos analizados en el estudio anterior, en este artículo se observó, a través de cuestionarios a sesenta y seis coordinadores que operan en las escuelas públicas de doce municipios paulistas, perteneciente a la mesorregión de Ribeirao Preto-SP, la necesidad de replantear la acción de coordinador pedagógico y el proceso de formación continua del servicio, las HTPCs.

Palabras clave: Coordinador pedagógico, Horas de Trabajo Colectivo Pedagógico (HTPCs), Formación continua, Resignificación.

Abstract: This article aims to expose some results of a master's research that aimed to analyze the limitations, possibilities and challenges regarding the performance of the pedagogical coordinator as an articulating agent in the continuing education of elementary school teachers. The article, in addition to providing definitions about the role of the pedagogical coordinator, analyzes how this professional works in the implementation of the processes of training developed in the Collective Pedagogical Work Schedule (HTPCs). In this context, it is highlighted that the professional career of the pedagogical coordinator is permeated by obstacles and that 
the educational system still has flaws regarding the pedagogical practice of this professional. The methodological path of the research involved descriptive and exploratory studies, in order to understand the reality of the pedagogical coordination in the schools defined for field research, with regard to its regulatory and practical-theoretical role. From the data analyzed in a previous study, this article observed, through questionnaires applied to sixty-six pedagogical coordinators working in municipal schools in twelve municipalities, belonging to the mesoregion of Ribeirão Preto, SP, the need to reframe the performance of the pedagogical coordinator, as well as the process of continuing in-service training of teachers, the HTPCs.

Keywords: Pedagogical coordinator, Collective Pedagogical Work Schedule (HTPC), Continuing in-service training, Resignification.

\section{INTRODUCCIÓN}

La formación continua en servicio ha sido la manera de mejorar la formación de los maestros en el entorno escolar. Desarrollada bajo la dirección de un coordinador pedagógico, la misma, según Nóvoa (2002) debe promover el pensamiento crítico, reflexivo y de autoformación participativa. Por lo tanto, es necesario que los maestros estén motivados y conscientes de la importancia de este proceso para su vida personal y profesional.

Dada la importancia de este proceso para los maestros de la escuela primaria, este artículo destaca cómo es, en la práctica, la actuación del coordinador pedagógico durante las HTPCs, y cómo estas se han establecido en la red municipal de docencia paulista. Según el comunicado de la Coordinadora de Estudios y Normas Pedagógicas de la Secretaría del Estado de San Pablo del 06 de febrero de 2009르. las HTPCs se caracterizan principalmente como espacio para la formación continua de educadores, propulsando momentos privilegiados de estudio, discusión y reflexión curricular y la mejora de la práctica docente; trabajo colectivo de carácter estrictamente pedagógico, dirigido para la discusión, el seguimiento y la evaluación de la propuesta educativa de la escuela y el rendimiento escolar del estudiante; las HTPCs deben ser planificadas y organizadas por el maestro coordinador de cada segmento de la educación primaria y secundaria en sintonía con todo el equipo directivo de la escuela, con el fin de integrar a todos los maestros del respectivo segmento, objeto de coordinación.

Este artículo es el resultado de la tesis Coordinador Pedagógico: Entre los desafíos de la actuación y la formación continua en servicio de los profesores de escuelas municipales del Estado de San Pablo (Lino, 2017), cuyo objetivo general fue dar a conocer cómo se desarrollan las relaciones de formación continua en el entorno escolar, analizar las tareas y desafíos relacionados con el trabajo del coordinador pedagógico, además de comprobar los límites y posibilidades que tiene y afronta la escuela pública municipal, en cuanto a la autonomía de construcción y desarrollo de proyectos de formación continua de sus maestros.

Por lo tanto, los datos que aquí se presentan fueron recuperados de la investigación realizada, a través de un protocolo de investigación que aplicó cuestionarios semiestructurados a sesenta y seis coordinadores activos en las escuelas públicas, en doce municipios que pertenecen a la mesorregión de Ribeirao Preto, SP, teniendo como principio una muestra no probabilística. Acerca de la selección de los municipios, se optó por incorporar de diferentes tamaños y que no adoptan un sistema educativo propio, lo que implica que siguen las instrucciones y las disposiciones legales de las escuelas del estado de San Pablo. Por lo tanto, en la presente investigación se utilizaron estudios descriptivos de carácter exploratorio con el fin de comprender la realidad del coordinador pedagógico en las escuelas investigadas. 
Consideramos que investigar, describir y analizar cómo el coordinador pedagógico ha trabajado en el entorno escolar puede resultar pertinente para los estudios que se inscriben en el campo de la educación, ya que los cambios que surgen constantemente en la sociedad requieren directivas apropiadas con el fin de guiar y desarrollar las prácticas de enseñanza de forma actualizada, prudente y reflexiva.

Para el desarrollo de la investigación, la base teórica fue construida en base a documentos oficiales, tales como la Ley de Directrices y Bases de la Educación y Referencias para la Formación de los Maestros (Brasil, 2002) y en trabajos ya publicados sobre el desempeño del coordinador: Almeida (2004), Nóvoa (2002), Garrido (2001), Souza (2004), Orsolon (2001), Gatti (2008), Placco y Almeida (2004), Tardif (2010), Imbernón (2004), Sacristán (1998), Giroux (1997), entre otros.

Según los estudios de Gatti (2008), en los últimos años del siglo XX

La educación continua fue colocada como una profundización y avance en la formación de profesionales. Esta necesidad se incorporó también a los sectores profesionales de la educación, que requirieron el desarrollo de políticas nacionales o regionales en respuesta a problemas propios de nuestro sistema educativo (Gatti, 2008, p. 58, traducción propia).

Por lo tanto, el propósito de este artículo asume como tema no sólo el papel del coordinador pedagógico en el proceso de formación continua, sino también su carácter relevante para la organización de la vida escolar en términos educativos, sociales y políticos. De este modo se reconoce la necesidad urgente de replantear el trabajo del coordinador pedagógico, así como de las HTPCs, con el fin de superar los obstáculos que comprometen la actuación de este profesional.

\section{El Coordinador pedagógico y las HTPCs en el Estado de San Pablo: definiendo SUS FUNCIONES}

Actualmente, el espacio dedicado al trabajo colectivo entre docentes de las escuelas públicas del estado de São Paulo se denomina HTPCs (Horas de trabajo pedagógico colectivo). De acuerdo con la legislación, este espacio debe ser desarrollado en la unidad escolar, por los profesores y el Coordinador Pedagógico y/o Director de la escuela.

Respecto al trabajo del coordinador pedagógico, la Secretaría de Educación del Estado de San Pablo emitió en el año 2007 la Resolución $N^{\circ}$ 88/2007, con la finalidad de determinar las atribuciones del Coordinador Pedagógico en todas las escuelas de su red de educación. Durante el mismo año, esa Resolución tuvo tres complementaciones, (con la complementación de las Resoluciones $\mathrm{N}^{\circ} 89$ y 90/2007) y modificaciones posteriores (Resoluciones SE N ${ }^{\circ} 53 / 2010,08 / 2011$ y 42/2012) definiendo las atribuciones específicas del Coordinador Pedagógico para los años iniciales y finales de la escuela primaria y secundaria y ampliando la responsabilidad de ese profesional en relación a otras áreas de atención educativa, como, por ejemplo, ofrecer apoyo a la organización de acciones culturales que estimulen el intercambio cultural, la integración participativa y la socialización.

En la red estatal de enseñanza, la Resolución SE N 75/2014, consolidada en el Programa de EducaciónCompromiso de San Paulo, define las atribuciones considerando los Coordinadores Pedagógicos, en palabras de Almeida, Souza y Placco (2016), como "los principales gestores de la política de Estado". Con respecto a las atribuciones, la Resolución SE N ${ }^{\circ} 75 / 2014$, con énfasis en las prácticas de gestión democrática y participativa, asegura de acuerdo con el artículo 5 , apartado 10, letra a) que "la participación proactiva de todos los docentes, en las horas de trabajo colectivo docente, promueve situaciones de orientación sobre prácticas docentes para el seguimiento y evaluación de propuestas de trabajo programadas" (SÃO PAULO, 2014).

En cuanto a la educación continua en el ámbito escolar, cabe mencionar que esta iniciativa surgió en el contexto paulista, de 1983 a 1988, a través del Decreto $\mathrm{N}^{\circ} 21.833$, de 1983, cuando el gobierno instituyó el Ciclo Básico de Educación Primaria, configurando el derecho a la formación continua del profesorado, dentro de la carga del trabajo del docente. La falta de estructura y efectividad de esta iniciativa de educación 
continua ha sufrido cambios, dando lugar a HTP (Hora de Trabajo Pedagógico). La función sufrió más modificaciones hasta que alcanzó la nomenclatura actual de HTPC, como explica Begnami,

\begin{abstract}
En 1988, con la implementación de la Jornada Única para estudiantes y docentes del ciclo básico, se amplió el concepto de HTP con el fin de establecer el lugar para su realización, además de asegurar la formación individual y colectiva de los docentes. En 1995, en un nuevo intento por superar el fracaso escolar, se implementó el Proyecto Educativo de la Escuela Estándar, mediante el Decreto No. 34.036 del 22/10/1991, con el objetivo de modernizar los procesos educativos, con la implementación paulatina de nuevas tecnologías y así recuperar la calidad de la enseñanza. Sin embargo, esta medida generó descontento con los docentes, ya que el decreto garantizó horas de trabajo pedagógico (HTP) en la jornada laboral solo para el 10\% (diez por ciento) de los docentes de la red de enseñanza. Con la reorganización de las Escuelas Públicas de San Pablo, a final de 1995, surgió el cargo de Profesor Coordinador Pedagógico y, en 1996, la Coordinación de Estudios y Normas Pedagógicas (CENP) publica la Ordenanza No. 01 de 08/05/1996, que instituye el trabajo pedagógico colectivo [...] En 1998, HTP, gana una nueva nomenclatura, Horario de Trabajo Pedagógico Colectivo (HTPC), con el propósito de garantizar momentos de formación colectiva dentro de la jornada laboral (Begnami, 2013, pp. 45-46, traducción propia).
\end{abstract}

Lo dispuesto en la dicha Portaria de la Coordinación de Estudios y Normas Pedagógicas - CENP N 01 de 1996, determinó que las HTPCs deberían ser realizadas en la Unidad Escolar por los profesores y por el profesor-coordinador pedagógico, con las siguientes finalidades:

articular los diferentes segmentos de la escuela para la construcción e implementación del trabajo pedagógico, fortalecer la Unidad Escolar como instancia privilegiada para la mejora de su proyecto pedagógico y (re) planificar y evaluar las actividades del aula, en vista de los lineamientos comunes que la escuela pretende para mejorar el proceso de enseñanza-aprendizaje. (SÃO PAULO, 1996, traducción propia).

De acuerdo con las definiciones y propósitos de las HPTCs, mencionadas anteriormente, corresponde al coordinador pedagógico, con el debido apoyo de los respectivos gestores, planificar y estimular el desarrollo de las actividades colectivas de la escuela. Esa misma legislación estableció, como objetivos de las HTPCs: articular las acciones educativas realizadas por los diferentes segmentos de la escuela, buscando la mejoría del proceso de enseñanza y aprendizaje; identificar las alternativas pedagógicas que existen para la reducción de los índices de evasión y repetición; posibilitar la reflexión sobre la práctica del profesor; favorecer el intercambio de experiencias; promover el perfeccionamiento individual y colectivo de los educadores; realizar un seguimiento y evaluar, de forma sistemática, el proceso de enseñanza-aprendizaje.

En cada reunión deben ser registradas en actas las actividades realizadas, como los temas trabajados, además de sus aspectos positivos y negativos, con la intención de evaluar los resultados obtenidos y colaborar en la toma de decisiones en relación a la planificación y a la continuidad del trabajo.

En resumen, el coordinador pedagógico integra el equipo de gestión escolar, junto con el equipo pedagógico, y debe ayudar en la organización de las diversas prácticas administrativas y pedagógicas diarias.

De acuerdo con Domingues:

La coordinación pedagógica es aún una actividad reciente, cuya profesión se está reelaborando debido a la especificidad de esta actuación, que se sustenta en diferentes sistemas de enseñanza, debido a la diversidad cultural de la escuela, y la pluralidad de los planes de estudio de la formación inicial y continua de este profesional (Domingues, 2009, p. 197).

La figura del coordinador pedagógico en las escuelas brasileñas todavía se considera reciente, ya que esta posición surgió con las transformaciones sufridas en la educación entre los años 70 y 90, cuando hubo transformaciones sociales, políticas y económicas. Como lo demuestra Pereira (2019), el coordinador pedagógico, antes de tener este nombre, tenía la función de supervisar la educación, controlar y supervisar las prácticas pedagógicas. Y es a partir de 1980, con el proceso de redemocratización de la enseñanza, que esta forma de intervenir será cuestionada. Sin embargo, fue sólo desde la Ley de Directrices y Bases de la Educación Nacional (Ley 9.394/1996) que la coordinación pedagógica atravesó un proceso de redefinición, dejando de lado su función más ligada a la supervisión y control para pasar a la de articulador de las propuestas pedagógicas y asumir la responsabilidad de la educación continua de los docentes de la escuela en la que se inserta el coordinador pedagógico. 
En el análisis de los estudios sobre las prácticas pedagógicas y la función del coordinador pedagógico, hemos observado que la formación continua es un proceso indispensable para la enseñanza de la práctica. Birgin (2012), al referirse a la formación continua, afirma que las cuestiones que atañen al sistema educativo de hoy también son parte de los problemas que constituyen la agenda de la formación: la construcción de lo común, las transformaciones de la enseñanza, los vaivenes de la autoridad, la transmisión de la cultura, entre otros.

Por lo tanto, al investigar la actuación del coordinador pedagógico, se comprobó que, desde el inicio de la década de 1990, la academia y los órganos oficiales del Brasil han fomentado una discusión en relación al ambiente escolar como un lugar de formación, buscando rescatar esos momentos colectivos con esta finalidad. En este recorte histórico, por así decirlo, existe una conquista por parte de los integrantes del magisterio oficial del estado de San Pablo, expresado por aportes legales como las Horas de Trabajo Pedagógico Colectivo.

Las municipalidades paulistas durante su proceso de organización en el campo educativo han contemplado en sus planes de estudio del magisterio y de cargos y salarios, el horario colectivo como un momento de perfeccionamiento. Desde entonces, e impulsadas por las políticas públicas de formación, se ha establecido la función del desarrollo profesional del profesor en las redes de enseñanza y en las escuelas.

Sin embargo, el papel de coordinador pedagógico no siempre es entendido en el alcance de sus funciones por la comunidad escolar y, en muchos casos, se confunde con el ayudante de dirección por motivos burocráticos, o consultado ante emergencias de los maestros, además de ser el responsable de la disciplina de los estudiantes.

Además de estas aristas vinculadas a su responsabilidad pedagógica, el coordinador también enfrenta las contradicciones presentes en los grupos sociales que terminan obstaculizando el trabajo colectivo, lo que requiere un profundo conocimiento de las relaciones humanas y la complejidad de los diversos grupos que forman la escuela, como estudiantes, maestros, padres, personal de apoyo y equipo técnico, compuesto por el director y los coordinadores.

Según Placco y Almeida (2004) el papel del coordinador pedagógico implica:

abordar la planificación, el desarrollo profesional y la formación del educador, las relaciones sociales e interpersonales existentes en la escuela es abordar la complejidad de lo humano, la formación de un ser humano que puede ser sujeto de transformación de sí mismo y de la realidad, llevando a cabo este entrenamiento él mismo, como resultado de la intencionalidad (Placco y Almeida, 2004, p. 59, traducción propia).

Por lo tanto, para llevar a cabo sus funciones de conformidad con las atribuciones que se le confieren, el coordinador pedagógico debe tener una formación inicial y continua, tanto en el aspecto teórico como en el aspecto práctico de la actividad educativa, ya que se espera que sea un investigador, con el fin de despertar el interés de los maestros y el deseo de estudio por los conocimientos que puedan ayudarlos en su práctica pedagógica.

En esta perspectiva, estamos de acuerdo con Orsolon (2001), cuando el autor afirma que: "El coordinador media el conocimiento, el saber-hacer, el saber-ser y actuar del docente" (p. 22).

Como se ve, en el proceso de formación continua hay muchos aspectos que intervienen, y, es importante no separar las dimensiones del saber y del saber-hacer. Al explorar y compartir el conocimiento durante las HTPCs, el coordinador actúa como mediador entre la teoría y la práctica, contribuyendo significativamente a la formación docente, sin embargo, hay que señalar que el proceso de autonomía intelectual, emocional, social y moral, se realiza de hecho, sólo cuando hay un fortalecimiento de la actuación del coordinador como formador, cuando se concibe su identidad profesional, a la luz de los momentos que exploran la capacidad reflexiva y crítica de los involucrados en el proceso. Además, como argumenta Lopez,

Podemos concluir que el tema de la formación docente tiene una gran complejidad, involucrando numerosas condiciones que van desde la propia constitución del profesionalismo y identidad docente, pasando por políticas públicas para la educación, alcanzando el aspecto relacional inherente el contexto escolar. Es necesario acciones integradas, con miras a espacio, tiempo y propuesta de formación dentro de las escuelas, lo que requiere macro y micro movimientos, políticas públicas y procesos colaborativos en las instituciones (Lopez, 2004, p. 146). 
En resumen, podemos decir que promover una educación de calidad, que garantice el aprendizaje para todos, razón de ser de la escuela, es una tarea de las más complejas y para su implementación requiere del compromiso, la cooperación y, sobre todo, el profesionalismo basado en el conocimiento.

Es en este contexto que la función del Coordinador Pedagógico se torna relevante en el ámbito escolar, asumiendo un rol articulador y ejecutor de las acciones educativas, especialmente en las HTPCs, con el objetivo de transformarlas en ambientes propicios para el desarrollo de la práctica pedagógica del equipo docente.

\section{Algunas imposiciones de LA ACCión DEL COORDiNADOR PEDAGógico}

En este artículo presentamos algunos aspectos que permean el trabajo del coordinador pedagógico. La recopilación de los datos para la tesis de maestría (Lino, 2017) destacan, a través de las opiniones de los coordinadores pedagógicos que respondieron al protocolo de investigación, aspectos de fragilidad en el proceso de educación continua en servicio en las escuelas que participaron en esta investigación, dado que muchos maestros no experimentan efectivamente los momentos de capacitación.

La investigación utilizó como instrumentos metodológicos la descripción y recolección de datos e informaciones a través de un cuestionario a sesenta y seis coordinadores pedagógicos, aplicados en un período de dos meses. Los participantes definidos para este estudio fueron profesionales vinculados a la Educación Fundamental de siete escuelas, ubicadas en doce municipios pertenecientes a la región geográfica de Ribeirao Preto, San Pablo. Sabiendo que los municipios pueden tener sistemas estructurados de enseñanza o redes de educación (sector público), para esa investigación privilegiamos siete municipios que están vinculadas, en su organización, al Estado de San Pablo. Aquellos municipios que optaron por el sistema propio (municipal) en su organización de enseñanza, no fueron incluidos como participantes de la investigación, tomando en cuenta que utilizan diferentes programas de formación continua en servicio, lo que inviabilizaría el propósito y la delimitación del presente estudio. En lo que respecta al protocolo de investigación, se elaboró un cuestionario compuesto por treinta y tres preguntas, siendo veintitres cerradas y diez abiertas, que posibilitaron a los participantes exponer libremente sus puntos de vista.

Los cuestionarios llegaron hasta los respondientes vía e-mail, o los entregamos personalmente, obteniendo un $93 \%$ de respuestas, siendo un porcentaje muy favorable para el alcance de los objetivos de la investigación.

Los datos obtenidos fueron organizados en tablas y, a partir de los resultados alcanzados, se procedió a su análisis teniendo como base a Bardin (2004) que define como: "una operación de clasificación de los elementos constitutivos de un conjunto, por diferenciación y luego por reagrupación, según el género, con criterios previamente definidos y o definidos a posteriori con el análisis del material” ( pp. 111-112), ya que las respuestas discursivas presentadas por los profesionales solicitaban una interpretación más detallada, teniendo en cuenta aquello que subyace al texto, como así también las inferencias.

Con referencia al tiempo de actuación como coordinadores pedagógicos, el $29 \%$ se encuentra en esa función hace menos de un año; el $22 \%$ entre uno y cinco años; el $41 \%$ entre seis y diez años; el $4 \%$ entre once y quince años y el $4 \%$ restante hace más de quince años.

Ante la pregunta sobre si participan en cursos de formación continua, de los sesenta y seis coordinadores pedagógicos que respondieron, el 62\% afirmó participar de los cursos que se brindan sobre su área de actuación específicamente. Esta pregunta fue elaborada con la finalidad de conocer si los coordinadores pedagógicos con formación en nivel de posgrado participan más, menos o con la misma frecuencia de cursos promovidos por el Estado de San Pablo o por el Municipio donde ejercen su función.

Según Imbernón (2000) la práctica docente debe estar impregnada de "conocimientos, habilidades y actitudes" (p. 39), que le permitan desarrollarse como un profesional reflexivo o investigador. En otras palabras, ser reflexivo significa tener la capacidad de pensar seriamente en su práctica docente, aprender a 
interpretar y comprender la realidad social, con el fin de buscar la manera de transformarla. De acuerdo con Libâneo (2011):

Los docentes, ante las nuevas realidades y la complejidad de los conocimientos que actualmente implican su formación profesional, necesitarían una formación teórica más profunda, capacidad operativa en los requerimientos de la profesión, propósitos éticos para afrontar la diversidad y diferencia cultural, además, obviamente, a la indispensable corrección en salarios, condiciones laborales y práctica profesional (Libâneo, p.77-78, traducción propia).

La necesidad de la actuación del coordinador pedagógico para el proceso de formación continua de los maestros es un hecho evidente. Al comienzo de nuestra investigación percibimos que las funciones realizadas por estos profesionales son muy importantes tanto para el desarrollo de los conocimientos de los maestros, como en la organización de diversas prácticas cotidianas ligadas a lo administrativo y pedagógico.

En esta perspectiva, Franco (2003, p. 124, traducción propia) explica que:

El proceso formativo requiere de procedimientos científicos, acciones coordinadas por profesionales pedagógicos, metodología propia, para construir un cuerpo de referencias, conocimientos, procedimientos, que puedan llevar a cabo esta nueva línea de investigación, ya no como una tendencia educativa, sino como una forma de reconstruir coherentemente caminos para la educación, superando problemas históricos que derivan en problemas de fracaso escolar, insuficiencia escolar y desvalorización de la pedagogía.

Nuestra investigación también muestra que el trabajo del coordinador pedagógico, aun visto como un proceso de carácter reciente, se encuentra condicionado por la organización institucional en la que se inscribe y por las políticas públicas, y menos por la formación académica que posee. En este sentido, contar o no con carrera de posgrado, no es una condición relevante para la participación de los coordinadores en cursos de formación continua para su área.

Otro aspecto importante se refiere al hecho de que el $51 \%$ de los coordinadores tiene poca experiencia en este rol, es decir, menos de cinco años. Sin embargo, el 33\% de los entrevistados informó que sólo participan de los cursos de educación continua que ofrece el Ministerio de Educación, lo que significa que un tercio de los coordinadores no buscan cursos que los actualice o califique para mejorar su práctica profesional. Esto resulta preocupante, ya que la función del coordinador pedagógico es, de acuerdo con Santoyo (1981):

propiciar el aprendizaje, de donde se derivan otras funciones como son: proponer el programa de estudios, observar y ofrecer retroalimentación sobre los cambios que se produzcan en la conducta del grupo, propiciar un ambiente favorable para el trabajo intelectual, procurar la comunicación y autodependencia del grupo, así como asesorar y evaluar las actividades de aprendizaje. Procederemos a definir al coordinador siempre en relación con las tareas y funciones. (Santoyo, p. 7, traducción propia).

En función de esto, Geglio (2004) agrega "es importante que el coordinador pedagógico lleve a cabo su acción en el seguimiento de las actividades de los docentes en el aula” (p. 118, traducción propia), dado que esto crea posibilidades para que los problemas que surjan en ese contexto se discutan y analicen desde una perspectiva diferenciada e integral. Sin embargo, uno de los entrevistados reconoce, a propósito de la función del coordinador pedagógico en la escuela, ciertas preocupaciones muy comunes en las actividades de este profesional:

Actualmente, es muy difícil actuar de manera confiable, de acuerdo con nuestro verdadero rol. La mayoría de las veces somos Bomberos: ¡apagamos incendios! El papel real queda en "otro" plano. La formación continua de los maestros que creo que es el principal objetivo del Coordinador Pedagógico no siempre es posible y aceptada. (Coordinador de Pedagógico de una de las escuelas investigadas) (Lino, 2017, p. 67).

Por otro lado, la falta de conocimiento de mi parte, por una formación inadecuada, genera inseguridad en el tratamiento de algunos temas, los cuales deben definirse para que respondan a los intereses y necesidades de todos los docentes, para que puedan participar con más implicación y sin las quejas habituales. (Coordinador Pedagógico de una de las escuelas investigadas) (Lino, 2017, p. 74). 
En palabras de los coordinadores, se destacan los aspectos negativos de la función de este profesional, es decir, las múltiples tareas que caen bajo la responsabilidad de la coordinación y reconocer que el proceso de formación no siempre es considerado como importante por los maestros, debido a la falta de formación inicial específica.

Refiriéndose al exceso de atribuciones del coordinador pedagógico, entre los entrevistados, el $70 \%$ ha colocado la excesiva cantidad de demandas como un factor negativo, lo que dificulta el desarrollo de acciones durante las HTPCs en las escuelas en las que trabajan. Para Garrido (2001) “[...] el maestro coordinador encuentra obstáculos para realizar su actividad. Lo atropellan las urgencias y necesidades de la rutina escolar. Como figura nueva y poco tradicional en la estructura institucional, sus funciones aún son poco entendidas y mal definidas" (p. 11, traducción propia). Por su parte, Souza (2004) establece que:

[el coordinador pedagógico] tendrá que aprender a vivir un eterno cuestionamiento: sobre los problemas y sus soluciones, sobre la propia forma de pensar y la del otro, para integrar nuevas suposiciones y concepciones. Y debe hacerlo junto con el grupo, sin renunciar al diálogo, porque todo el grupo debe aceptar la complejidad para que se cambie la forma de actuar y pensar la educación (Souza, 2004, p. 110, traducción propia).

Según Placco y Silva (2000), la formación continua en la educación es "un proceso complejo y multidimensional, que gana materialidad en múltiples espacios/actividades, no restringiéndose a cursos y/ o capacitaciones [...]" (p. 27). Sin embargo, cuando se le preguntó si los cursos de educación continua que ofrece el Ministerio de Educación son adecuados a su realidad, la mayoría de los coordinadores participantes en la investigación respondieron que están satisfechos con las directrices y el proceso de formación continua promovidos por el Estado. Sin embargo, sólo el 29\% de los coordinadores consideran que este tipo de cursos les dan apoyo teórico para el desarrollo de su actividad docente. Ya el 35\% de los entrevistados dijo que estos cursos no resultan suficientes para mejorar su práctica pedagógica.

Por lo tanto, esta reducida formación teórica y práctica mencionada por algunos coordinadores, conduce a su vez, a inferir como consecuencia una escasa formación de estos profesionales, que se profundiza por la falta de apoyo de los administradores, tanto de la escuela como de los gestores públicos, en particular el personal de las Secretarias de Enseñanza Municipal. Preocupados por este aspecto, este estudio alrededor de la actuación de este profesional busca destacar la importancia que asume el coordinador pedagógico como un investigador activo y dedicado al estudio y análisis de la realidad educativa, con el fin de tener una sólida base teórica que contribuya al proceso de formación continua en las escuelas donde trabaja.

\section{HTPCs y LOS OBSTÁCULOS DE LA FORMACIÓN CONTINUA EN SERVICIO}

De acuerdo a los dictados proporcionadas por la Coordinación Estatal de Normas Pedagógicas (São Paulo, 1997), a través de la Ordenanza $N^{\circ} 1 / 1996$, regulada por la Ley Complementaria $\mathrm{N}^{\circ} 836 / 1997$ y notificación de 20 de enero de 2008 y 06 de febrero de 2009, las HTPCs deben desarrollarse dentro de la escuela por los maestros, con la orientación del coordinador pedagógico, sujeta a la siguiente organización: por cada 10 a 27 horas de trabajo en el aula, los maestros deben dedicar 2 horas a estas reuniones, y dedicar 3 horas cuando tenga una jornada de trabajo en el aula de 28 a 33 horas, con el objetivo principal de crear un espacio de discusión y capacitación para fortalecer el proyecto político-pedagógico de la escuela. En el caso de las redes educativas municipales elegidas para la investigación, las propuestas de capacitación en servicio cumplen con los requisitos de la legislación educativa del Estado de San Pablo, especialmente con respecto a los deberes del coordinador pedagógico.

Es durante este trabajo colectivo y continuo que, según Garrido (2001), los maestros deben crear propuestas educativas para afrontar los desafíos de su escuela. Sin embargo, como parte de nuestra investigación, encontramos que la formación continua realizada durante las HTPCs no se produce en algunas escuelas analizadas ${ }^{2}$ de forma continua y contextual y tampoco se lleva a cabo en el lugar donde el coordinador 
realiza su función. Algunos coordinadores afirmaron que "no hay momentos en que todos los maestros participan juntos, por lo que no hay HTPCs en mi escuela, lo que explica que el mismo maestro trabaja en más de una escuela el mismo día" (Lino, 2017, p. 72). Otro dato relevante es que la duración de las HTPCs se establece en función del número de horas que cada uno de los maestros ejerce en la escuela, aspecto que es reconocido por el $65 \%$ de los coordinadores como uno de los factores que interfieren con el buen desarrollo de las HTPCs, como se expone en el siguiente gráfico.

Los obstáculos para el desarrollo de las Horas de Trabajo Pedagógico Colectivo - HTPCs

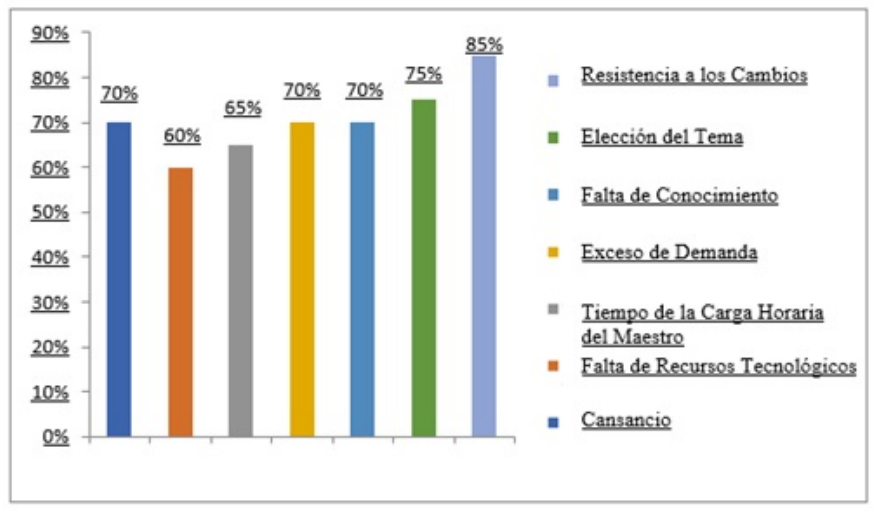

Fuente: Análisis de los datos recopilados. Elaboración propia (2020).

Otra dificultad señalada por los coordinadores (70\%), es el cansancio de los maestros, ya que las HTPCs se llevan a cabo después de la finalización de las clases, y que repercute en la participación de las sesiones.

Vinculado a esto, existe aún otro obstáculo señalado por el $85 \%$ de los coordinadores pedagógicos, que se refiere a la dificultad de cambiar las concepciones ya existentes sobre el proceso de enseñanza y aprendizaje desde un marco teórico y así transformar su práctica pedagógica, convirtiéndose en más creativos, interesante y relevante, como afirma acertadamente uno de los encuestados:

En la diversidad de opiniones e intereses, el docente no siempre está completamente direccionado en servir a los estudiantes de la mejor manera posible. Encontramos resistencia por parte de algunos profesores que piensan que llevan mucho tiempo enseñando y que ya lo saben todo "sin necesidad de aprender más para poder enseñar mejor". (Coordinador Pedagógico de una de las escuelas investigadas) (Lino, 2017, p. 76).

Otro aspecto señalado por el $75 \%$ de los coordinadores como un elemento que complica su trabajo en las HTPCs, es la definición de los temas que se tratarán en las reuniones, procurando que sean de interés para todos los maestros y que su estudio y discusión contribuyan, de hecho, para la mejora de su formación docente. En cuanto a este aspecto, algunos coordinadores se han posicionado explicando que:

Junto con la Dirección del Colegio, los temas se definen a partir de lo que se percibe como las necesidades diarias de la rutina del maestro que necesitan ser aclaradas. A partir de la definición del tema, existe una extensa investigación sobre el material al respecto y otros recursos relevantes al mismo como forma de enriquecerlo. (Coordinador Pedagógico de una de las escuelas investigadas). (Lino, 2017, p. 77).

[La definición de la agenda y los temas para las HTPCs] están de acuerdo con las necesidades verificadas en las evaluaciones diagnósticas aplicadas a los estudiantes, con los índices presentados en las evaluaciones externas, con las orientaciones recibidas del equipo pedagógico municipal y con las necesidades de los propios maestros, cuando solicitan un tema concreto en el que quieran profundizarse (Coordinador Pedagógico de una de las escuelas investigadas). (Lino, 2017, p. 77).

Uno de los entrevistados afirmó que "si la planificación está bien hecha y el contenido bien preparado, de acuerdo con las necesidades de los maestros, no habrá dificultades para llevar a cabo las HTPCs" (Lino, 2017, p. 76). Estamos de acuerdo con esta posición, porque un plan bien diseñado contribuirá eficazmente a la 
motivación de los educadores para involucrarse en el proceso de educación continua en servicio, sin embargo, no es una simple acción de logro, porque como destaca Sacristán (2000),

Planificar la práctica es algo más amplio que cumplir con los objetivos y contenidos del currículo, porque supone preparar las condiciones para su desarrollo, ya que también lo cumple. Es darse cuenta de las condiciones en las que se llevará a cabo el currículum, y eso significa establecer un puente para moldear las ideas a la realidad, un vínculo entre las intenciones y la acción (Sacristán, 2000, p. 282, traducción propia).

Los coordinadores pedagógicos investigados señalaron que existe un déficit en cuanto a su formación inicial, el $70 \%$ dijo que este es un factor que crea inseguridad sobre todo para trabajar los temas propuestos por los maestros. Tardif (2010), en relación con la formación de los maestros, tanto inicial como continua, argumenta que el conocimiento de la experiencia tiene una importancia estratégica, pues "[...] para los profesores, el conocimiento adquirido a través de la experiencia profesional es la base de su competencia. Es a partir de ellos que los docentes juzgan su formación previa o su formación a lo largo de su carrera" (p. 48). Para Santiago (2004) "el dominio del cuerpo teórico, actualizado por la reflexión colectiva, puede dar a los docentes autonomía de acción, creatividad, posibilidades para la construcción de instrumentos didácticos, alternativas metodológicas [...] en resumen, capacidad de gestión" (p. 163). Sin embargo, los coordinadores investigados dicen que tratan de prepararse de la mejor manera posible para las tareas que la escuela y la sociedad le demandan. También entienden que las HTPCs ocurren en días diferentes de aquellos en los que los maestros enseñan, de contar con diversos materiales, dispositivos multimedia, el resultado de la actuación podría ser más significativo. En este sentido, la encuesta también puso de relieve que la falta de recursos tecnológicos se convierte en un obstáculo para el buen funcionamiento de la formación de los maestros, considerándose un problema por el $60 \%$ de los coordinadores.

Sin embargo, los posicionamientos citados a continuación, registran la autovalorización del verdadero papel de este profesional, ya que demuestran el compromiso asumido con el mejoramiento de la calidad de la enseñanza en la escuela donde trabajan:

A pesar de mi inexperiencia, me he dedicado mucho y aprendido cada día. Creo que todos los temas elegidos hasta ahora han sido de gran beneficio para los profesores y para mí. Ser coordinadora me ha dado muchas novedades y también otra mirada a la rutina escolar. (Coordinador Pedagógico de una de las escuelas investigadas) (Lino, 2017, p. 78)

Trabajo duro para cumplir con mis propias expectativas y aunque mi equipo es pequeño, la mayor parte del tiempo, incluso con tan poca gente, me siento importante en mi rol de ayudar al profesor a mejorar sus clases y su proceso de enseñanza y aprendizaje. (Coordinador de Pedagógico de una de las escuelas investigadas) (Lino, 2017, p. 78).

Como se ve, la formación continua es de hecho un proceso, según Placco e Silva (2000) complejo y multideterminado. Además de los conflictos personales, el coordinador pedagógico también enfrenta exigencias de su desempeño real, impuestas por el contexto o el sistema adoptado por la escuela en la que trabaja. En este sentido, Almeida (2004) establece que: "El Coordinador Pedagógico debe considerar que existirán obstáculos para el desempeño de sus actividades. Debe predecir que se verá afectado por las emergencias y necesidades de la rutina escolar. Debe ser consciente de que sus funciones aún son poco conocidas y definidas" (p. 30).

Sin embargo, a pesar de los obstáculos que enfrentan los coordinadores en su práctica diaria, todavía encuentran la fuerza para buscar mejores alternativas educativas y pedagógicas para sus escuelas y esto fue destacado como un factor positivo por la investigación. Otro aspecto positivo se refiere a la participación de la mayoría de los gestores en la planificación, incluyendo la preparación de las agendas y la discusión de los temas, así como en la evaluación del trabajo desarrollado durante las HTPCs, mediante el análisis de los informes presentados por los coordinadores al final de cada uno de estos momentos de la formación continua.

Aun así, reflexionar sobre la urgencia del proceso de formación continua en el servicio y el compromiso del coordinador pedagógico a que las HTPCs se conviertan, de hecho, en tiempos de formación. En otras palabras, existe una necesidad urgente del coordinador pedagógico de comprender la relevancia de su práctica 
pedagógica que, en el diseño de Placco y Almeida (2004) es: "planificar e interferir en el desarrollo profesional y la formación docente, incluido el estudio y la crítica de teorías [...]" (p. 58), acercarse a ellos a partir de su práctica, permitiendo y fomentando las contradicciones entre los fundamentos teóricos y la realidad de la rutina escolar.

\section{CONSIDERACIONES FINALES}

El sistema educativo brasileño presenta aún muchas dificultades en relación a la formación continua de los maestros, trasladando esta tarea a las escuelas, los administradores y sus coordinadores. Esta investigación, permitió conocer a través de las escuelas municipales de Sao Paulo, que aún queda un largo camino por recorrer para que se produzca la formación efectiva de los maestros durante las HTPCs, en tanto se identifican una serie de factores negativos que interfieren en el desarrollo de sesiones continuas de formación de los maestros en las escuelas.

Este artículo permite demostrar que aún persisten dificultades en el proceso de formación continua en el servicio, algunas de los cuales se relacionan con la sobrecarga de trabajo de los maestros, la resistencia al cambio, así como el cansancio. Junto a esto, hay otros factores, tales como el exceso de actividades que el coordinador pedagógico tiene que resolver y la falta de formación específica, que impactan de manera negativa en el desarrollo profesional.

Además, es necesario que el coordinador pedagógico comprenda la relevancia de su práctica pedagógica, que según Nogueira (2008) debe “ir más allá de los conocimientos teóricos, porque para acompañar el trabajo pedagógico y estimular a los docentes se necesita percepción y sensibilidad para identificar las necesidades de estudiantes y docentes [...]" (p. 1).

Por lo tanto, alertamos sobre la necesidad de políticas públicas que valoren la práctica docente, a través de la mejora de sus condiciones laborales, propuestas de formación continua en el servicio que puedan ser consideradas como un agregado a su desarrollo profesional, y no como una acumulación de trabajo obligatorio, ya que durante las HTPCs se encuentran cansados debido a la carga de trabajo diaria.

De acuerdo con los obstáculos presentados en este artículo, refiriéndose al rol del Coordinador Pedagógico durante el proceso de formación continua de los docentes en servicio, destacamos la necesidad de una redefinición de las Horas de Trabajo Pedagógico Colectivo - HTPCs, considerando que los desafíos en el ámbito educativo son muchos, creemos que el momento de la formación continua es la principal vía para promover cambios relevantes en el ámbito escolar, especialmente en la práctica docente.

De esta forma, de acuerdo en que la formación de los maestros es un proceso que debe extenderse al entorno de trabajo a través de acciones de formación continua, se asume la necesidad de un fuerte vínculo entre lo que promueve la formación y los entornos de trabajo típicos de estos profesionales, es decir, HTPCs, que debe centrarse en las necesidades que enfrentan los maestros en la vida escolar. Y que, en tiempos de formación, el ambiente escolar es un espacio democrático de ideas, propuestas y producción de conocimiento. En otras palabras, promover que las instancias de formación continua propicien un espacio de reflexión, individual y colectivo sobre las prácticas pedagógicas, donde el coordinador pueda asumir un rol de coordinación y facilitador del proceso de enseñanza y aprendizaje.

\section{BibLIOGRAFÍA}

Almeida, L. R. de. (2004). Um dia na vida de um coordenador pedagógico de escola pública. En Placco, V. M. N. de S. e Almeida, L. R. de. (Ed.). O coordenador pedagógico e o cotidiano da escola. $2^{a}$ ed. São Paulo: Edições Loyola

Almeida, L. R., Souza, V. L. T. e Placco, V. M. G. N. (2016). Legislado versus executado: análise das atribuiçóes formativas do coordenador pedagógico. Estudos em Avaliação Educacional,27(64), pp. 70-94. Recuperado de: h ttp://publicacoes.fcc.org.br/index.php/eae/article/view/3647/3156 
Bardin, L. (2004). Análise de Conteúdo. Lisboa: Edições 70.

Begnami, M. L.V. (2013). Formação Continuada: o HTPC como espaçopara a autonomia formativa. (Tesis de maestría). Centro Universitário Salesiano de São Paulo, São Paulo.

Birgin, A. (2012). Más allá de la capacitación. Debates acerca de la formación de los docentes en ejercicio. Buenos Aires: Paidós.

Brasil. (1996).Lei de Diretrizes e Basespara a Educação. Leiño 9.394/96, de 20 de dezembro de 1996. Brasília: Ministério da Educação.

Brasil. (2002). Referenciais para Formação de Professores. Brasília: Ministério da Educação. Secretaria de Educação Fundamental.

Domingues, I. (2009). O coordenador pedagógico e o desafio da formação contínua do docente na escola. (Tesis Doctoral). Faculdade de Educação da Universidade de São Paulo, São Paulo.

Garrido, E. (2001). Espaço de Formação Contínua para o Professor-Coordenador. In Bruno, E. B. G., Almeida, L. R. de e Christov, L. H. da S. (Ed.) O coordenador pedagógico e a formação docente. $2^{\mathrm{a}}$ ed. São Paulo: Ediçóes Loyola.

Gatti, B. A. (2008). Análise das políticas públicas para formação continuada no Brasil, na última década. Revista Brasileira de Educação, 13(37), pp. 57-70. ISSN 1809-449X. Recuperado de https://www.redalyc.org/articulo .oa? $\mathrm{id}=27503706$

Franco, M. A. S (2003). Pedagogia como ciência da educação. Campinas, SP: Papirus.

Geglio, P. C. (2004). O papel do coordenador pedagógico na formação do professor em serviço. En Placco, V. M. N de S. e Almeida, L, R. de. (Ed.), O coordenador Pedagógico e o cotidiano da escola. 2 ed. São Paulo: Ediçôes Loyola.

Imbernón, F. (2004). Formação Docente e Profissional: Formar-se para a mudança e a incerteza. $4^{a}$ ed. São Paulo: Cortez.

Libâneo, J. C. (2011). Adeus professor, adeus professora?: novas exigências educacionais e profissão docente. $13^{a}$ ed. São Paulo: Cortez.

Lino, J. P. (2017). Coordinador Pedagógico: Entre los desafios de la actuación y la formación continua en servicio de los profesores de escuelas municipales del Estado de San Paulo. (Tesis de maestria). Universidad Nacional de La Plata, La Plata, Argentina.

Lopez, A. C. T. (2004). Formação de professores: reflexão e Emancipação. Nuances: Estudos sobre Educação. UNESP, 10(11-12). Recuperado de: https://revista.fct.unesp.br/index.php/Nuances/article/view/394/429

Nogueira, V. S. (2008). O papel do coordenador pedagógico. Brasil Escola. Recuperado de: https://educador.brasiles cola.uol.com.br/trabalho-docente/o-papel-coordenador-pedagogico.htm

Nóvoa, A. (2002). Formação de professores e trabalho pedagógico. Lisboa: Educa

Orsolon, L. A. M. (2001). O coordenador/formador como um dos agentes de transformação da/na escola. En Almeida, L. R. de. e Placco, V. M. N. de S. O coordenador pedagógico e o espaço da mudança. São Paulo: Edições Loyola.

Pereira, L. C. (2019). Coordenador Pedagógico. Info Escola. Recuperado de: https://www.infoescola.com/educacao /coordenador-pedagogico/

Placco, V. M. N. S e Silva, S. H. S. (2000). A formação do professor: reflexões, desafios e perspectivas. En Bruno, E. B., Almeida, L. R. e Christov, L. H. (Ed.), O coordenador pedagógico e a formação docente. São Paulo: Loyola, pp. 25-32.

Placco, V.M.N de S. e Almeida, L, R. de. (2004). O coordenador Pedagógico e o cotidiano da escola. 2a ed. São Paulo: Edições Loyola.

Santiago, A. R. F. (2004). Projeto Político-Pedagógico da Escola: Desafio à Organização dos Educadores. En VEIGA, I. P. A., Projeto Político-Pedagógico da Escola: Uma Construção Possível. 17 a ed. Campinas, São Paulo: Papirus Editora.

Sacristán, J. G. (2000). O Curriculo: Uma Reflexão sobre a Prática. Porto Alegre: Artmed.

São Paulo. (1983). Decreto n. 21.833, de 28 de dezembro de 1983. São Paulo: Assembleia Legislativa do Estado de São Paulo. Recuperado de: https://www.al.sp.gov.br /repositorio/legislacao/decreto/1983/decreto-21833 28.12.1983.html.

São Paulo. (1997). Lei Complementar no 836, de 30 de dezembro de 1997. São Paulo. 
São Paulo. (2007). Resolução SE n. 88, de 19 de dezembro de 2007. São Paulo: Secretaria de Educação.

São Paulo. (2007). Resolução SE n. 89, de 19 de dezembro de 2007. São Paulo: Secretaria de Educação.

São Paulo. (2007). Resolução SE n. 90, de 19 de dezembro de 2007. São Paulo: Secretaria de Educação.

São Paulo. (2014). Resolução SE n. 75, de 31 de dezembro de 2014. São Paulo: Secretaria de Educação.

São Paulo. (1983). Decreto n. 21.833, de 28 de dezembro de 1983. Recuperado de: https://www.al.sp.gov.br/ norma/56465.

São Paulo. (1997). Horas de trabalho pedagógico coletivo - HTPC. Portaria CENP no $1 / 96$ Lei Complementar $\mathrm{n}^{\circ}$ 836/97. Recuperado de: http://educacaotiete.sp.gov.br/wp-content/uploads/2014/11/1-Comentario-Portari a-CENP-01-96.pdf

São Paulo. (1996). Portaria CENP, 1 de maio de 1996. São Paulo: Diário Oficial do Estado.

Santoyo, R. (1981). Algunas reflexiones sobre la coordinación en los grupos de aprendizaje. Perfiles Educativos, (11), pp. 3-19. ISNN: 0185-2698. Recuperado de: https://docenciaiep.files.wordpress.com/2016/03/algunas_reflex iones.pdf

Souza, V.L. T. de. (2004). O coordenador pedagógico e o atendimento à diversidade. In Placco, V.M.N de S. e Almeida, L, R. de. (Ed.), O coordenador Pedagógico e o cotidiano da escola. 2a ed. São Paulo: Edições Loyola.

Souza, G. R. de. (2013). Horas de Trabalho Pedagógico Coletivo (HTPCs): espaços de formação contínua e de produção de saberes docentes? (Tesis de maestría). Universidade Estadual Paulista Júlio de Mesquita Filho, Faculdade de Ciências e Tecnologia, São Paulo. Recuperado de: https://repositorio.unesp.br/handle/11449/92363

Tardif. M. (2010). Saberes Docentes e Formação Profissional. 10 ed. Rio de Janeiro: Vozes.

\section{Notas}

1 Recuperado de http://www.sindicatoapase.com.br/ler-legislacao/101-comunicado-cenp--de-06-02-2009.

2 Entre los sesenta y seis coordinadores Pedagógicos encuestados, nueve dijeron que las HTPCs no suceden en sus escuelas y que la educación continua se lleva a cabo a través de cursos ofrecidos por el Municipal de Educación y Cultura - SMEC, o empresas contratadas para ello (Lino, 2017, p. 76). 